\title{
Current and future observations of Near Earth Objects with SALT and RSS
}

\section{David E. Trilling*}

South African Astronomical Observatory, P.O. Box 9, Observatory, Cape Town 7935, South Africa; University of the Western Cape, Bellville, Cape Town 7535, South Africa; and Northern Arizona University, P.O. Box 6010, Flagstaff, AZ 86011, United States

E-mail: david.trilling@nau.edu

\section{Amanda A. Sickafoose}

South African Astronomical Observatory, P.O. Box 9, Observatory, Cape Town 7935, South Africa

E-mail: amanda@saao.ac.za

We report here results from a SALT/RSS pilot programme to observe newly-discovered Near Earth Objects. These small asteroids (as small as tens of metres) are only accessible to large ground-based facilities for a few nights after discovery. Our programme utilizes Target of Opportunity observations (priority 0 in the SALT queue) to obtain compositional information about these small bodies. In this paper we present first results from this pilot programme as well as discuss some current challenges in carrying out this programme with SALT.

SALT Science Conference 2015

1-5 June 2015

Stellenbosch Institute of Advanced Study, South Africa

\footnotetext{
* Speaker.
} 


\section{Introduction}

Near Earth Objects (NEOs) are small Solar System bodies whose orbits bring them close to the Earth's orbit. NEOs are delivered to near-Earth space from elsewhere in the Solar System [1], so studying NEOs as they fly by the Earth is akin to in situ measurements of small bodies throughout the Solar System, at a fraction of the cost of a spacecraft mission. Furthermore, NEOs are our nearest neighbours in space, and sometimes hit the Earth (as happened in Chelyabinsk, Russia, in February, 2013), so the study of NEO properties has both scientific and practical motivations.

NEOs are typically discovered by optical ground-based survey programmes that have a limiting magnitude of around $\mathrm{V}=21 \mathrm{mag}$. At present, the discovery rate is around 1000 NEOs per year, much higher than the characterization rate of less than 100 per year. Therefore, our overall knowledge of the properties of the NEO population is decreasing with time as the fraction of NEOs with measured physical properties decreases with time. In particular, the smallest NEOs, which fade rapidly, are particularly poorly observed and understood. To reverse this trend, new, efficient methods of measuring NEO properites must be deployed. Furthermore, such observations must be made rapidly after discovery, as NEOs may fade by one magnitude or more per week. The traditional observational approach of observing targets weeks or months after discovery is not viable for NEOs.

The primary spectral features used to identify asteroid taxonomies and therefore implied compositions are continuum slope and the presence of mineral-caused absorption features, which are typically on the order of 0.2 microns wide [2]. To classify asteroid spectra taxonomically therefore only very low resolution spectra are needed - around $\mathrm{R} \sim 25$ or so is usually sufficient. This in turn implies that (a) most spectrographs have native resolutions that are far higher than needed for asteroid work and (b) because we will consequently heavily bin the spectra, intrinsically low signal-to-noise spectra can be acquired in the native resolution.

In 2014 and 2015 we carried out a pilot programme to use the Robert Stobie Spectrograph on SALT in Target of Opportunity ( $\mathrm{ToO}$ ) mode to obtain spectra and therefore compositions of small NEOs and to determine how effective SALT could be as a rapid response NEO characterization facility. Here we report observations of two small NEOs in this ToO mode, and discuss the future possibility of continuing to use SALT as a critical component for NEO characterization.

\section{Observations and results}

During the 2014B semester we observed two small NEOs with SALT/RSS. 2014 YM9 was discovered on 20 Dec 2014 by the Pan-STARRS survey. It has a Solar System absolute magnitude of 19.3 mag, implying a size around 350 metres. We observed this asteroid on 30 Jan 2015, when its apparent V magnitude was around 16 mag. Few NEOs have apparitions this bright, so this was a good test case for our first observation in this programme: a newly discovered very bright target that would allow us to test our acquisition and analysis strategies before pushing to very faint magnitudes.

2015 GY was discovered on 10 Apr 2015, also by the Pan-STARRS survey. It has a Solar System absolute magnitude of $21.7 \mathrm{mag}$, implying a size around 125 metres. We observed this asteroid on 22 Apr 2015, when its apparent V magnitude was around 19 mag. This target was 
somewhat more difficult to acquire at the telescope, due to its relative faintness, though once the data were obtained the reduction was no more difficult than for the much brighter 2014 YM9.

Both targets were observed in the same way, with 900 seconds total integration time split over five individual exposures. The slit was aligned along the motion vector of the asteroid so that the telescope was tracked at its usual sidereal rates, and the asteroids traveled in the along-slit direction during the $\sim 1000$ seconds (exposures plus overhead) on source. If either asteroid had been very fast moving, a dither in the along-slit direction would have been required to keep the asteroid in the slit. The targets were observed in the longslit mode of the Robert Stobie Spectrograph [3, 4], using grating pg 300 , which offers the lowest native resolution (and broadest wavelength coverage).

For each asteroid, a nearby Solar analogue star (G2V, or similar) was observed. This standard star serves two purposes. The first is that the standard observations can be used to monitor and remove atmospheric contamination from the spectra. The second is that we are interested in measuring the intrinsic reflectance spectrum of an asteroid, so we must divide the observed spectrum by a spectrum of the Sun (or similar). Each Solar analogue star observation was identical to the asteroid observation except that the total integration time for the (bright) standard star was six seconds total.

The data were reduced following the PySALT pipeline [5], through bias subtraction; gain correction; cross-talk fixes; flat-fielding; cosmic-ray cleaning; wavelength calibration; removing the sky; and extracting the spectra by summing across the spatial region containing the asteroid signal. We normalized each spectrum to unity at $5500 \AA$, and divided each spectrum by the appropriate Solar analogue. Finally, these analogue-divided spectra were rebinned from the native resolution of 350 to a final resolution of around 25 across the spectral range 4800-10700 $\AA$.

The resulting final binned spectra are shown in Figure 1. We also show in Figure 1 the archetypal spectra [2] for S- and C-type asteroids, which are the two dominant asteroid taxonomic (and hence composition) types. Both of the asteroids that we observed in this pilot programme have spectra that are clearly consistent with S-type spectra due to their red slope in the 5000-7500 $\AA$ region and their suggested absorption feature at around $9000 \AA$; these two features (red slope, feature near $9000 \AA$ ) are the two primary defining features of S-type spectra. In contrast, C-type spectra are essentially flat across this wavelength range.

\section{Discussion}

Both of the small NEOs that we observed have S-type spectra, which is suggestive of a silicate (rocky) composition. It has recently been shown [6] that around 50\% of NEOs in the size range we are probing here are S-type bodies, so our small-number statistics of $2 / 2$ bodies being S-type is consistent with the larger sample. Of course, a larger sample would also reveal C-type asteroids, and potentially other types, all of which should be detectable and identifiable with this method.

More broadly, we conclude that this pilot project was successful: the technique of using sidereal tracking with asteroids moving along the slit is a viable way of taking data, and allows for acceptable sky subtraction. The Solar analogue observations were also feasible, and allowed for proper calibration. 


\section{Conclusions and future work}

Our primary conclusion is that SALT with RSS works successfully for rapid response characterization of small NEOs. SALT has two niches that could profitably be exploited for this project. The first is that the primary NEO characterization instrument currently in use is SpeX [7] on NASA's 3-meter IRTF on Maunakea in Hawai'i. Typically, at that facility, objects fainter than $\mathrm{V}=18$ mag are difficult to observe in this "reconnaissance spectroscopy" mode. Therefore, SALT, with its substantially larger aperture, would be best utilized by observing targets with $\mathrm{V}>18$ mag. The second advantage is geographic. Most NEO characterization telescopes are in Chile and the western United States - approximately 180 degrees longitude from Sutherland, South Africa. NEOs can have very short windows in which they are bright enough to be observed spectroscopically, so there may easily be targets that are observable only with SALT and no other facility. In the future, the near infrared arm of RSS will provide additional power for this project, as asteroid spectral features in the infrared provide even more diagnostic power than in the optical.

The largest current NEO spectroscopy programmes are the MIT-UH-IRTF Joint Campaign for NEO Spectral Reconnaissance (http://smass.mit.edu/minus.html) and the MANOS programme [8], each of which catalogues spectra for several tens of objects per year. For SALT/RSS to make a significant contribution in this rapid-response field, a sample of similar size would be required. At roughly one hour per target, SALT could make a significant contribution to this field with the relatively small allocation of around 25 hours per year.

There are several issues that prevent SALT from being readily used for this kind of rapidresponse programme at present. First, non-sidereal tracking is not a commissioned mode on the telescope. Hence, the rate of motion is limited to relatively slow-moving NEOs so that no along-slit dithers are required. However, slow-moving NEOs are those that are relatively far from the Earth, which means that they must be relatively large in order to be bright enough to be observed. In other words, the rate limitation corresponds to a lower limit on the size that can be observed. Second, in some cases acquiring the NEO and placing it in the RSS slit was difficult - in part because the positional uncertainties of NEOs can often be tens of arcseconds - so our sensitivity limit is functionally set by target acquisition, not the photons in the asteroid's spectrum. Finally, we found that triggering $\mathrm{ToO}$ observations for moving objects required extensive manual configuration and support from SALT staff. Such an effort would clearly not be sustainable for a programme that is frequently observing ToO targets. None of these issues is impossible to solve, though, and there may be a time in the future when SALT can be used regularly as a unique resource to measure NEO compositions.

\section{Acknowledgments}

We thank Michael Mommert for his target selection code; Christian Hettlage for extensive help getting our targets into the SALT queue; Encarni Romero Colmenero for providing work-arounds so that our targets could be observed promptly; the SALT support astronomers who managed to observe our targets; and Steve Crawford for providing data reduction routines. 


\section{References}

[1] Bottke et al. 2002, Debiased Orbital and Absolute Magnitude Distribution of the Near-Earth Objects, Icarus, 156, 399

[2] Demeo et al. 2009, An extension of the Bus asteroid taxonomy into the near-infrared, Icarus, 202, 160

[3] Burgh et al. 2003, Prime Focus Imaging Spectrograph for the Southern African Large Telescope: optical design, SPIE, 4841, 1463

[4] Kobulnicky et al. 2003, Prime focus imaging spectrograph for the Southern African large telescope: operational modes, SPIE, 4841, 1634

[5] Crawford et al. 2010, PySALT: the SALT science pipeline, SPIE, 7737, 25

[6] Mommert et al. 2015, Rapid-Response Spectrophotometric Characterization of Near-Earth Objects using UKIRT, AJ, submitted

[7] Rayner et al. 2003, SpeX: A Medium-Resolution 0.8-5.5 Micron Spectrograph and Imager for the NASA Infrared Telescope Facility, PASP, 115, 362

[8] Moskovitz et al. 2015, The Mission Accessible Near-Earth Object Survey (MANOS) - Science Highlights, published in IAU General Assembly, 22, 55616 


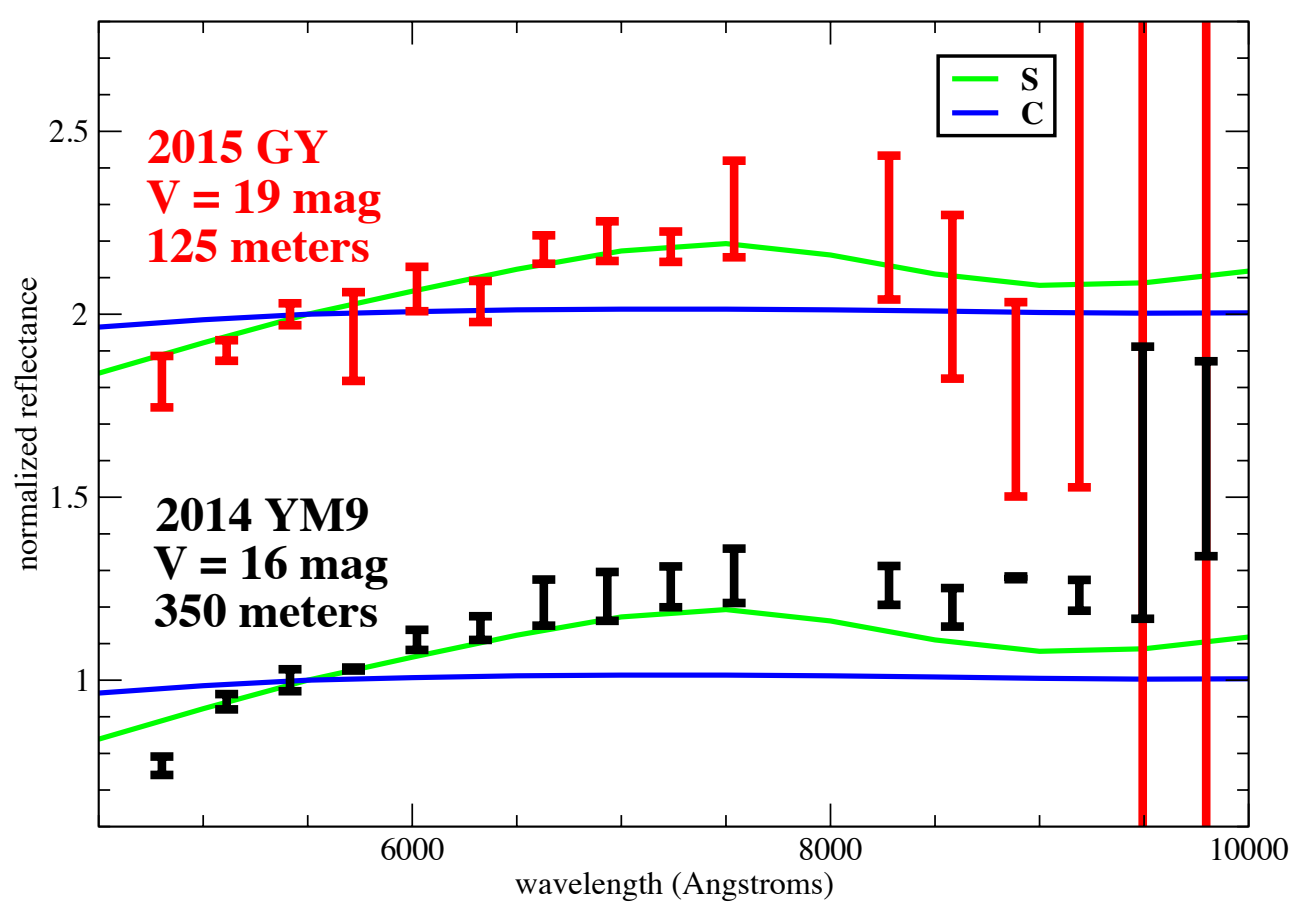

Figure 1: SALT/RSS spectra of two small NEOs acquired during 2014 and 2015 (black and red lines). For comparison, representative spectra (green and blue lines) are shown for the two most dominant asteroid taxonomic classes, from [2]. The SALT data have been heavily binned since only broad features are needed to establish taxonomy. The observed NEOs are consistent with S-type asteroids (green line), having a red slope and an absorption feature near $9000 \AA$. All spectra are normalized to unity at $5500 \AA$; the reflectance spectrum of $2015 \mathrm{GY}$ and its comparison spectra are all offset upward by 1.0 for clarity. There is no measurement at $\sim 8000 \AA$ because there is a chip gap at that wavelength location. The bright target $(\mathrm{V}=16 \mathrm{mag})$ has very high SNR and an asteroid taxonomy is easily identified. The SNR for the fainter target (V=19 mag) is worse, especially at the longest wavelengths, but is still sufficient for taxonomic identification. 